\title{
Strong convergence theorems for fixed point problems of a nonexpansive semigroup in a Banach space
}

\author{
Xin Wang, Changsong Hu* and Jinlin Guan
}

\section{"Correspondence:}

huchang1004@yahoo.com.cn

School of Mathematics and

Statistics, Hubei Normal University,

Huangshi, 435002, China

\begin{abstract}
In this paper, we study the implicit and explicit viscosity iteration schemes for a nonexpansive semigroup in a reflexive, strictly convex and uniformly smooth Banach space which satisfies Opial's condition. Our results improve and generalize the corresponding results given by Yao et al. (Fixed Point Theory Appl. 2013, doi:10.1186/1687-1812-2013-31) and many others.
\end{abstract}

MSC: $47 \mathrm{H} 05 ; 47 \mathrm{H} 10 ; 47 \mathrm{H} 17$

Keywords: nonexpansive semigroup; fixed point; reflexive and strictly convex Banach space; uniformly smooth; Opial's condition; sunny nonexpansive retraction

\section{Introduction}

Let $E$ be a real Banach space, and let $K$ be a nonempty, closed and convex subset of $E$. A mapping $T: K \rightarrow K$ is called nonexpansive if

$$
\|T x-T y\| \leq\|x-y\|, \quad \forall x, y \in K
$$

One parameter family $S:=\{T(s): 0 \leq s<\infty\}$ is said to be a nonexpansive semigroup from $K$ into $K$ if the following conditions are satisfied:

(1) $T(0) x=x$ for all $x \in K$;

(2) $T(s+t)=T(s) T(t)$ for all $s, t \geq 0$;

(3) $\|T(t) x-T(t) y\| \leq\|x-y\|, \forall x, y \in K$ and $t \geq 0$;

(4) for each $x \in K$, the mapping $T(\cdot) x$ from $[0, \infty)$ into $K$ is continuous.

Let $F(S)$ denote the common fixed point set of the semigroup $S$, i.e., $F(S):=\{x \in K$ : $T(s) x=x, \forall s>0\}$. It is known that $F(S)$ is closed and convex.

A continuous operator of the semigroup $S$ is said to be uniformly asymptotically regular (u.a.r.) on $K$ if for all $h \geq 0$ and any bounded subset $C$ of $K, \lim _{s \rightarrow \infty} \sup _{x \in C} \| T(h) T(s) x-$ $T(s) x \|=0$ (see [1]).

Approximation of fixed points of nonexpansive mappings by a sequence of finite means has been considered by many authors (see [2-6]). In 2013, Yao et al. [7] introduced two new algorithms for finding a common fixed point of a nonexpansive semigroup $\{T(s)\}_{s \geq 0}$ in Hilbert spaces and proved that both approaches converge strongly to a common fixed point of $\{T(s)\}_{s \geq 0}$.

C2013 Wang et al.; licensee Springer. This is an Open Access article distributed under the terms of the Creative Commons Attribution License (http://creativecommons.org/licenses/by/2.0), which permits unrestricted use, distribution, and reproduction in any medium, provided the original work is properly cited. 
Theorem 1.1 [7] Let $C$ be a nonempty closed convex subset of a real Hilbert space $H$. Let $S=\{T(s)\}_{s \geq 0}: C \rightarrow C$ be a nonexpansive semigroup with $\operatorname{Fix}(S) \neq \emptyset$. Let $\left\{\gamma_{t}\right\}_{0<t<1}$ and $\left\{\lambda_{t}\right\}_{0<t<1}$ be two continuous nets of positive real numbers such that $\gamma_{t} \in(0,1), \lim _{t \rightarrow 0} \gamma_{t}=1$ and $\lim _{t \rightarrow 0} \lambda_{t}=+\infty$. Let $\left\{x_{t}\right\}$ be the net defined in the following implicit manner:

$$
x_{t}=P_{C}\left[t\left(\gamma_{t} x_{t}\right)+(1-t) \frac{1}{\lambda_{t}} \int_{0}^{\lambda_{t}} T(s) x_{t} d s\right], \quad \forall t \in(0,1) .
$$

Then, as $t \rightarrow 0^{+}$, the net $\left\{x_{t}\right\}$ strongly converges to $x^{*} \in \operatorname{Fix}(s)$.

Theorem 1.2 [7] Let $C$ be a nonempty closed convex subset of a real Hilbert space $H$. Let $S=\{T(s)\}_{s \geq 0}: C \rightarrow C$ be a nonexpansive semigroup with $\operatorname{Fix}(S) \neq \emptyset$. Let $\left\{x_{n}\right\}$ be the sequence generated iteratively by the following explicit algorithm:

$$
x_{n+1}=\left(1-\beta_{n}\right) x_{n}+\beta_{n} P_{C}\left[\alpha_{n}\left(\gamma_{n} x_{n}\right)+\left(1-\alpha_{n}\right) \frac{1}{\lambda_{n}} \int_{0}^{\lambda_{n}} T(s) x_{n} d s\right], \quad \forall n \geq 0,
$$

where $\left\{\alpha_{n}\right\},\left\{\beta_{n}\right\}$ and $\left\{\gamma_{n}\right\}$ are sequences of real numbers in $[0,1]$ and $\left\{\lambda_{n}\right\}$ is a sequence of positive real numbers. Suppose that the following conditions are satisfied:

(i) $\lim _{n \rightarrow \infty} \alpha_{n}=0, \sum_{n=0}^{\infty} \alpha_{n}=\infty$ and $\lim _{n \rightarrow \infty} \gamma_{n}=1$;

(ii) $0<\liminf _{n \rightarrow \infty} \beta_{n} \leq \lim \sup _{n \rightarrow \infty} \beta_{n}<1$;

(iii) $\lim _{n \rightarrow \infty} \lambda_{n}=\infty$ and $\lim _{n \rightarrow \infty} \frac{\lambda_{n-1}}{\lambda_{n}}=1$.

Then the sequence $\left\{x_{n}\right\}$ generated by (1.3) strongly converges to a point $x^{*} \in \operatorname{Fix}(s)$.

In this paper, we study the convergence of the following iterative schemes in a reflexive, strictly convex and uniformly smooth Banach space which satisfies Opial's condition:

$$
\begin{aligned}
& x_{t}=Q_{K}\left[t\left(\gamma_{t} x_{t}\right)+(1-t) T\left(s_{t}\right) x_{t}\right], \quad \forall t \in(0,1), \\
& x_{n+1}=\left(1-\beta_{n}\right) x_{n}+\beta_{n} Q_{K}\left[\alpha_{n}\left(\gamma_{n} x_{n}\right)+\left(1-\alpha_{n}\right) T\left(s_{n}\right) x_{n}\right], \quad \forall n \geq 0 .
\end{aligned}
$$

Our work improves and generalizes many others. In particular, our results extend the main results of Yao et al. [7].

\section{Preliminaries}

Let $E$ be a real Banach space and $E^{*}$ be the dual space of $E$. The duality mapping $J: E \rightarrow 2^{E^{*}}$ is defined by

$$
(x)=\left\{f \in E^{*}:\langle x, f\rangle=\|x\|^{2}=\|f\|^{2}\right\} .
$$

By the Hahn-Banach theorem, $J(x)$ is nonempty.

Let $\operatorname{dim} E \geq 2$. The modulus of convexity of $E$ is the function $\delta_{E}:(0,2] \rightarrow[0,1]$ defined by

$$
\delta_{E}(\epsilon):=\inf \left\{1-\left\|\frac{x-y}{2}\right\|:\|x\|=\|y\|=1 ; \epsilon=\|x-y\|\right\} .
$$

$E$ is uniformly convex if $\forall \epsilon \in(0,2]$, there exists $\delta=\delta(\epsilon)>0$ such that if $x, y \in E$ with $\|x\| \leq 1,\|y\| \leq 1$ and $\|x-y\| \geq \epsilon$, then $\left\|\frac{x+y}{2}\right\| \leq 1-\delta$. Equivalently, $E$ is uniformly convex if 
and only if $\delta_{E}(\epsilon)>0, \forall \epsilon \in(0,2]$. $E$ is strictly convex if for all $x, y \in E, x \neq y,\|x\|=\|y\|=1$, we have $\|\lambda x+(1-\lambda) y\|<1, \forall \lambda \in(0,1)$.

Let $S(E)=\{x \in E:\|x\|=1\}$. The space $E$ is said to be smooth if

$$
\lim _{t \rightarrow 0}(\|x+t y\|-\|x\|) / t
$$

exists for all $x, y \in S(E)$. The norm of $E$ is said to be Fréchet differentiable if for all $x \in$ $S(E)$, the limit (2.3) exists uniformly for all $y \in S(E)$. $E$ is said to have a uniformly Gâteaux differentiable norm if for all $y \in S(E)$, the limit (2.3) is attained uniformly for all $x \in S(E)$. The norm of $E$ is said to be uniformly Fréchet differentiable (or uniformly smooth) if the limit (2.3) is attained uniformly for $x, y \in S(E) \times S(E)$.

It is well known that if $E$ is smooth, then $J$ is single-valued, which is denoted by $j$. And if $E$ has a uniformly Gâteaux differentiable norm, then $J$ is norm-to-weak* uniformly continuous on each bounded subset of $E$. The duality mapping $J$ is said to be weakly sequentially continuous if $J$ is single-valued and for any $\left\{x_{n}\right\} \in E$ with $x_{n} \rightarrow x, J\left(x_{n}\right) \rightarrow^{*} J(x)$. Gossez and Lami Dozo [8] proved that a space with a weakly continuous duality mappings satisfies Opial's condition. Conversely, if a space satisfies Opial's condition and has a uniformly Gâteaux differentiable norm, then it has a weakly continuous duality mapping.

Recall that if $C$ and $D$ are nonempty subsets of a Banach space $E$ such that $C$ is nonempty closed convex and $D \subset C$, the mapping $Q: C \rightarrow D$ is said to be sunny if

$$
Q(Q x+t(x-Q x))=Q x
$$

where $Q x+t(x-Q x) \in C$ for all $x \in C$ and $t \geq 0$.

A mapping $Q: C \rightarrow D$ is called a retraction if $Q x=x$ for all $x \in D$.

A subset $D$ of $C$ is called a sunny nonexpansive retraction of $C$ if there exists a sunny nonexpansive retraction from $C$ into $D$ (see $[9,10]$ ). It is well known that if $E$ is a Hilbert space, then a sunny nonexpansive retraction is coincident with the metric projection from $E$ onto $C$.

Proposition 2.1 [11] Let C be a closed convex subset of a smooth Banach space E. Let D be a nonempty subset of $C$. Let $Q: C \rightarrow D$ be a retraction, and let $J$ be the normalized duality mapping on $E$. Then the following are equivalent:

(1) $Q$ is sunny and nonexpansive.

(2) $\|Q x-Q y\|^{2} \leq\langle x-y, J(Q x-Q y)\rangle, \forall x, y \in C$.

(3) $\langle x-Q x, J(y-Q x)\rangle \leq 0, \forall x \in C, y \in D$.

Proposition 2.2 [12] Let $C$ be a nonempty closed convex subset of a strictly convex and uniformly smooth Banach space $E$, and let $T$ be a nonexpansive mapping of $C$ into itself with $F(T) \neq \emptyset$. Then the set $F(S)$ is a sunny nonexpansive retraction of $C$.

Lemma 2.3 [13] Let $K$ be a nonempty closed convex subset of a reflexive Banach space $E$ which satisfies Opial's condition, and suppose that $T: K \rightarrow E$ is nonexpansive. Then the mapping $I-T$ is demiclosed at zero, that is, $x_{n} \rightarrow x, x_{n}-T x_{n} \rightarrow 0$ implies $x=T x$.

Lemma 2.4 [14] Let $\left\{x_{n}\right\},\left\{y_{n}\right\}$ be two bounded sequences in a Banach space $E$ and $\beta_{n} \in$ $(0,1)$ with $0<\liminf _{n \rightarrow \infty} \beta_{n} \leq \limsup _{n \rightarrow \infty} \beta_{n}<1$. Suppose $x_{n+1}=\beta_{n} y_{n}+\left(1-\beta_{n}\right) x_{n}$ for all integers $n \geq 0$ and $\limsup _{n \rightarrow \infty}\left(\left\|y_{n+1}-y_{n}\right\|-\left\|x_{n+1}-x_{n}\right\|\right) \leq 0$. Then $\lim _{n \rightarrow \infty}\left\|x_{n}-y_{n}\right\|=0$. 
Lemma 2.5 [15] Let $\left\{a_{n}\right\}$ be a sequence of nonnegative real numbers satisfying the following relation:

$$
a_{n+1} \leq\left(1-\rho_{n}\right) a_{n}+\rho_{n} \sigma_{n}, \quad n \geq 0,
$$

where $\left\{\rho_{n}\right\}$ and $\left\{\sigma_{n}\right\}$ are sequences of real numbers such that

(i) $0<\rho_{n}<1$;

(ii) $\sum_{n=1}^{\infty} \rho_{n}=\infty$;

(iii) $\limsup _{n \rightarrow \infty} \sigma_{n} \leq 0$ or $\sum_{n=1}^{\infty}\left|\rho_{n} \sigma_{n}\right|$ is convergent.

Then $\lim _{n \rightarrow \infty} a_{n}=0$.

\section{Main result}

Theorem 3.1 Let E be a reflexive, strictly convex and uniformly smooth Banach space which satisfies Opial's condition, and let $K$ be a nonempty closed convex subset of $E$. Let $S=\{T(s): s \geq 0\}: K \rightarrow K$ be a uniformly asymptotically regular nonexpansive semigroup such that $F(S) \neq \emptyset$. Let $\left\{\gamma_{t}\right\}_{0<t<1}$ and $\left\{s_{t}\right\}_{0<t<1}$ be two continuous nets of positive real numbers such that $\gamma_{t} \in(0,1), \lim _{t \rightarrow 0} \gamma_{t}=1$ and $\lim _{t \rightarrow 0} s_{t}=+\infty$. Let $\left\{x_{t}\right\}$ be the net defined by

$$
x_{t}=Q_{K}\left[t\left(\gamma_{t} x_{t}\right)+(1-t) T\left(s_{t}\right) x_{t}\right], \quad \forall t \in(0,1) \text {. }
$$

Then, as $t \rightarrow 0^{+}$, the net $\left\{x_{t}\right\}$ converges strongly to a point $x^{*} \in F(S)$.

Proof Consider a mapping $W$ on $K$ defined by

$$
W x:=Q_{K}\left[t\left(\gamma_{t} x\right)+(1-t) T\left(s_{t}\right) x\right], \quad \forall t \in(0,1) .
$$

$\forall x, y \in K$, we have

$$
\begin{aligned}
\|W x-W y\| & \leq\left\|t \gamma_{t}(x-y)+(1-t)\left(T\left(s_{t}\right) x-T\left(s_{t}\right) y\right)\right\| \\
& \leq t \gamma_{t}\|x-y\|+(1-t)\|x-y\| \\
& =\left[1-\left(1-\gamma_{t}\right) t\right]\|x-y\| .
\end{aligned}
$$

Hence, $W$ is a contraction. So, it has a unique fixed point, denoted by $x_{t}$. That is,

$$
x_{t}=Q_{K}\left[t\left(\gamma_{t} x_{t}\right)+(1-t) T\left(s_{t}\right) x_{t}\right] \text {. }
$$

Therefore, the sequence $\left\{x_{t}\right\}$ defined by (3.1) is well defined.

Let $p \in F(S)$, then

$$
\begin{aligned}
\left\|x_{t}-p\right\| & =\left\|Q_{K}\left[t\left(\gamma_{t} x_{t}\right)+(1-t) T\left(s_{t}\right) x_{t}\right]-p\right\| \\
& \leq\left\|t \gamma_{t}\left(x_{t}-p\right)-t\left(1-\gamma_{t}\right) p+(1-t)\left(T\left(s_{t}\right) x_{t}-p\right)\right\| \\
& \leq t \gamma_{t}\left\|x_{t}-p\right\|+t\left(1-\gamma_{t}\right)\|p\|+(1-t)\left\|x_{t}-p\right\| \\
& =\left[1-\left(1-\gamma_{t}\right) t\right]\left\|x_{t}-p\right\|+t\left(1-\gamma_{t}\right)\|p\| .
\end{aligned}
$$


It follows that

$$
\left\|x_{t}-p\right\| \leq\|p\|
$$

Thus, $\left\{x_{t}\right\}$ is bounded, so is $\left\{T\left(s_{t}\right) u_{n}\right\}$.

Let $R=\|p\|$. It is clear that $\left\{x_{t}\right\} \subset B(p, R)$. Then $B(p, R) \cap K$ is a nonempty bounded closed convex subset of $K$ and $T(s)$-invariant. Since $\{T(s)\}$ is u.a.r. nonexpansive semigroup and $\lim _{t \rightarrow 0} s_{t}=\infty$, then for all $s>0$,

$$
\lim _{t \rightarrow 0}\left\|T(s)\left(T\left(s_{t}\right) x_{t}\right)-T\left(s_{t}\right) x_{t}\right\| \leq \lim _{n \rightarrow \infty} \sup _{x \in D}\left\|T(s)\left(T\left(s_{t}\right) x\right)-T\left(s_{t}\right) x\right\|=0,
$$

where $D$ is any bounded subset of $K$ containing $\left\{u_{n}\right\}$. Since

$$
\left\|x_{t}-T\left(s_{t}\right) x_{t}\right\| \leq t\left\|\gamma_{t} x_{t}-T\left(s_{t}\right) x_{t}\right\| \rightarrow 0,
$$

and

$$
\begin{aligned}
\left\|x_{t}-T(s) x_{t}\right\| & \leq\left\|x_{t}-T\left(s_{t}\right) x_{t}\right\|+\left\|T\left(s_{t}\right) x_{t}-T(s)\left(T\left(s_{t}\right) x_{t}\right)\right\|+\left\|T(s)\left(T\left(s_{t}\right) x_{t}\right)-T(s) x_{t}\right\| \\
& \leq 2\left\|x_{t}-T\left(s_{t}\right) x_{t}\right\|+\left\|T\left(s_{t}\right) x_{t}-T(s)\left(T\left(s_{t}\right) x_{t}\right)\right\| .
\end{aligned}
$$

Thus, for all $s>0$, we have

$$
\lim _{t \rightarrow 0}\left\|x_{t}-T(s) x_{t}\right\|=0
$$

Set $y_{t}=t\left(\gamma_{t} x_{t}\right)+(1-t) T\left(s_{t}\right) x_{t}$. Then $x_{t}=Q_{K} y_{t}$. By Proposition 2.1(2), we can get that

$$
\begin{aligned}
\left\|x_{t}-p\right\|^{2} & =\left\|Q_{K} y_{t}-Q_{K} p\right\|^{2} \\
& \leq\left\langle y_{t}-p, j\left(x_{t}-p\right)\right\rangle \\
& =t \gamma_{t}\left\langle x_{t}-p, j\left(x_{t}-p\right)\right\rangle-t\left(1-\gamma_{t}\right)\left\langle p, j\left(x_{t}-p\right)\right\rangle+(1-t)\left\langle T\left(s_{t}\right) x_{t}-p, j\left(x_{t}-p\right)\right\rangle \\
& \leq\left[1-\left(1-\gamma_{t}\right) t\right]\left\|x_{t}-p\right\|^{2}-t\left(1-\gamma_{t}\right)\left\langle p, j\left(x_{t}-p\right)\right\rangle .
\end{aligned}
$$

Thus

$$
\left\|x_{t}-p\right\|^{2} \leq-\left\langle p, j\left(x_{t}-p\right)\right\rangle, \quad \forall p \in F(S)
$$

Since $\left\{x_{t}\right\}$ is bounded and $E$ is reflexive, there exists a subsequence $\left\{x_{t_{n}}\right\}$ of $\left\{x_{t}\right\}$ such that $x_{t_{n}} \rightarrow x^{*}$. From (3.2), we have $x_{t_{n}}-T(s) x_{t_{n}} \rightarrow 0$ as $n \rightarrow \infty$. Since $E$ satisfies Opial's condition, it follows from Lemma 2.3 that $x^{*} \in F(S)$. From (3.3), we have

$$
\left\|x_{t_{n}}-p\right\|^{2} \leq-\left\langle p, j\left(x_{t_{n}}-p\right)\right\rangle, \quad \forall p \in F(S) .
$$

In particular, if we substitute $x^{*}$ for $p$ in (3.4), then we have

$$
\left\|x_{t_{n}}-x^{*}\right\|^{2} \leq-\left\langle x^{*}, j\left(x_{t_{n}}-x^{*}\right)\right\rangle .
$$


Since $j$ is weakly sequentially continuous from $E$ to $E^{*}$, it follows from (3.5) that

$$
\lim _{n \rightarrow \infty}\left\|x_{t_{n}}-x^{*}\right\|^{2} \leq \lim _{n \rightarrow \infty}-\left\langle x^{*}, j\left(x_{t_{n}}-x^{*}\right)\right\rangle=0 .
$$

Suppose that there exists a subsequence $\left\{x_{t_{m}}\right\}$ of $\left\{x_{t}\right\}$ such that $x_{t_{m}} \rightarrow \tilde{x}$. Then we have $\tilde{x} \in F(S)$ and

$$
\left\|x_{t_{m}}-p\right\|^{2} \leq-\left\langle p, j\left(x_{t_{m}}-p\right)\right\rangle, \quad \forall p \in F(S) .
$$

Since $x^{*}, \tilde{x} \in F(S)$, from (3.4) and (3.6), we have

$$
\left\|x_{t_{n}}-\widetilde{x}\right\|^{2} \leq-\left\langle\widetilde{x}, j\left(x_{t_{n}}-\widetilde{x}\right)\right\rangle,
$$

and

$$
\left\|x_{t_{m}}-x^{*}\right\|^{2} \leq-\left\langle x^{*}, j\left(x_{t_{m}}-x^{*}\right)\right\rangle .
$$

Now, in (3.7) and (3.8), taking $n \rightarrow \infty$ and $m \rightarrow \infty$, respectively. We get

$$
\left\|x^{*}-\widetilde{x}\right\|^{2} \leq-\left|\widetilde{x}, j\left(x^{*}-\tilde{x}\right)\right\rangle
$$

and

$$
\left\|\widetilde{x}-x^{*}\right\|^{2} \leq-\left\langle x^{*}, j\left(\widetilde{x}-x^{*}\right)\right\rangle .
$$

Adding up (3.9) and (3.10), we have

$$
\left\|x^{*}-\tilde{x}\right\|^{2} \leq 0 .
$$

We have proved that each cluster point of $\left\{x_{t}\right\}$ (as $t \rightarrow 0$ ) equals $x^{*}$. Thus $x_{t} \rightarrow x^{*}$ as $t \rightarrow 0$.

Remark 3.2 Theorem 3.1 improves and extends Theorem 3.1 of Yao et al. [7] in the following aspects.

(1) From a real Hilbert space to a reflexive, strictly convex and uniformly smooth Banach space which satisfies Opial's condition.

(2) $\frac{1}{\lambda_{t}} \int_{0}^{\lambda_{t}} T(s) x_{t} d s$ is replaced by $T\left(s_{t}\right) x_{t}$.

Theorem 3.3 Let E be a reflexive, strictly convex and uniformly smooth Banach space which satisfies Opial's condition, and let $K$ be a nonempty closed convex subset of $E$. Let $S=\{T(s): s \geq 0\}: K \rightarrow K$ be a uniformly asymptotically regular nonexpansive semigroup such that $F(S) \neq \emptyset$. Let $\left\{x_{n}\right\}$ be a sequence generated in the following iterative process:

$$
x_{n+1}=\left(1-\beta_{n}\right) x_{n}+\beta_{n} Q_{K}\left[\alpha_{n}\left(\gamma_{n} x_{n}\right)+\left(1-\alpha_{n}\right) T\left(s_{n}\right) x_{n}\right], \quad \forall n \geq 0,
$$

where $\left\{\alpha_{n}\right\},\left\{\beta_{n}\right\}$ and $\left\{\gamma_{n}\right\}$ are sequences of real numbers in $[0,1]$ satisfying the following conditions: 
(1) $\lim _{n \rightarrow \infty} \gamma_{n}=1, \sum_{n=1}^{\infty}\left(1-\gamma_{n}\right) \alpha_{n}=\infty, \lim _{n \rightarrow \infty} \alpha_{n}=0$.

(2) $0<\liminf _{n \rightarrow \infty} \beta_{n} \leq \limsup _{n \rightarrow \infty} \beta_{n}<1$.

(3) $h, s_{n} \geq 0$ such that $s_{n+1}=h+s_{n}$ and $\lim _{n \rightarrow \infty} s_{n}=\infty$.

Then $\left\{x_{n}\right\}$ converges strongly to $x^{*} \in F(S)$.

Proof Let $p \in F(S)$, we can get

$$
\begin{aligned}
\left\|x_{n+1}-p\right\| & =\left\|\left(1-\beta_{n}\right) x_{n}+\beta_{n} Q_{K}\left[\alpha_{n}\left(\gamma_{n} x_{n}\right)+\left(1-\alpha_{n}\right) T\left(s_{n}\right) x_{n}\right]-p\right\| \\
& \leq\left(1-\beta_{n}\right)\left\|x_{n}-p\right\|+\beta_{n}\left\|Q_{K}\left[\alpha_{n}\left(\gamma_{n} x_{n}\right)+\left(1-\alpha_{n}\right) T\left(s_{n}\right) x_{n}\right]-p\right\| \\
& \leq\left(1-\beta_{n}\right)\left\|x_{n}-p\right\|+\beta_{n}\left\|\alpha_{n} \gamma_{n}\left(x_{n}-p\right)-\alpha_{n}\left(1-\gamma_{n}\right) p+\left(1-\alpha_{n}\right)\left(T\left(s_{n}\right) x_{n}-p\right)\right\| \\
& \leq\left(1-\beta_{n}\right)\left\|x_{n}-p\right\|+\beta_{n}\left(\alpha_{n} \gamma_{n}\left\|x_{n}-p\right\|-\alpha_{n}\left(1-\gamma_{n}\right)\|p\|+\left(1-\alpha_{n}\right)\left\|x_{n}-p\right\|\right) \\
& =\left[1-\left(1-\gamma_{n}\right) \alpha_{n} \beta_{n}\right]\left\|x_{n}-p\right\|+\left(1-\gamma_{n}\right) \alpha_{n} \beta_{n}\|p\| \\
& \leq \max \left\{\left\|x_{n}-p\right\|,\|p\|\right\} \\
& \leq \max \left\{\left\|x_{0}-p\right\|,\|p\|\right\} .
\end{aligned}
$$

Hence, $\left\{x_{n}\right\}$ is bounded, so is $\left\{T\left(s_{n}\right) x_{n}\right\}$.

Set $y_{n}=Q_{K}\left[\alpha_{n}\left(\gamma_{n} x_{n}\right)+\left(1-\alpha_{n}\right) T\left(s_{n}\right) x_{n}\right]$ for all $n \geq 0$. Then $x_{n+1}=\left(1-\beta_{n}\right) x_{n}+\beta_{n} y_{n}$.

$$
\begin{aligned}
\left\|y_{n+1}-y_{n}\right\|= & \| Q_{K}\left[\alpha_{n+1}\left(\gamma_{n+1} x_{n+1}\right)+\left(1-\alpha_{n+1}\right) T\left(s_{n+1}\right) x_{n+1}\right] \\
& -Q_{K}\left[\alpha_{n}\left(\gamma_{n} x_{n}\right)+\left(1-\alpha_{n}\right) T\left(s_{n}\right) x_{n}\right] \| \\
\leq & \left\|\left[\alpha_{n+1}\left(\gamma_{n+1} x_{n+1}\right)+\left(1-\alpha_{n+1}\right) T\left(s_{n+1}\right) x_{n+1}\right]-\left[\alpha_{n}\left(\gamma_{n} x_{n}\right)+\left(1-\alpha_{n}\right) T\left(s_{n}\right) x_{n}\right]\right\| \\
= & \| \alpha_{n+1} \gamma_{n+1}\left(x_{n+1}-x_{n}\right)+\left(\alpha_{n+1} \gamma_{n+1}-\alpha_{n} \gamma_{n}\right) x_{n}+\left(1-\alpha_{n+1}\right) \\
& \times\left(T\left(s_{n+1}\right) x_{n+1}-T\left(s_{n+1}\right) x_{n}+T\left(s_{n+1}\right) x_{n}-T\left(s_{n}\right) x_{n}\right)+\left(\alpha_{n+1}-\alpha_{n}\right) T\left(s_{n}\right) x_{n} \| \\
\leq & \alpha_{n+1} \gamma_{n+1}\left\|x_{n+1}-x_{n}\right\|+\left|\alpha_{n+1} \gamma_{n+1}-\alpha_{n} \gamma_{n}\right|\left\|x_{n}\right\| \\
& +\left(1-\alpha_{n+1}\right)\left(\left\|x_{n+1}-x_{n}\right\|+\left\|T(h) T\left(s_{n}\right) x_{n}-T\left(s_{n}\right) x_{n}\right\|\right) \\
& +\left|\alpha_{n+1}-\alpha_{n}\right|\left\|T\left(s_{n}\right) x_{n}\right\| \\
= & {\left[1-\left(1-\gamma_{n+1}\right) \alpha_{n+1}\right]\left\|x_{n+1}-x_{n}\right\|+\left|\alpha_{n+1} \gamma_{n+1}-\alpha_{n} \gamma_{n}\right|\left\|x_{n}\right\| } \\
& +\left(1-\alpha_{n+1}\right)\left\|T(h)\left(s_{n}\right) x_{n}-T\left(s_{n}\right) x_{n}\right\|+\left|\alpha_{n+1}-\alpha_{n}\right|\left\|T\left(s_{n}\right) x_{n}\right\| .
\end{aligned}
$$

So,

$$
\begin{aligned}
& \left\|y_{n+1}-y_{n}\right\|-\left\|x_{n+1}-x_{n}\right\| \\
& \leq-\left(1-\gamma_{n+1}\right) \alpha_{n+1}\left\|x_{n+1}-x_{n}\right\|+\left|\alpha_{n+1} \gamma_{n+1}-\alpha_{n} \gamma_{n}\right|\left\|x_{n}\right\| \\
& \quad+\left(1-\alpha_{n+1}\right)\left\|T(h)\left(s_{n}\right) x_{n}-T\left(s_{n}\right) x_{n}\right\|+\left|\alpha_{n+1}-\alpha_{n}\right|\left\|T\left(s_{n}\right) x_{n}\right\| .
\end{aligned}
$$

Since $\{T(s): s \geq 0\}$ is uniformly asymptotically regular and $\lim _{n \rightarrow \infty} s_{n}=\infty$, it follows that

$$
\lim _{n \rightarrow \infty}\left\|T(h) T\left(s_{n}\right) x_{n}-T\left(s_{n}\right) x_{n}\right\| \leq \lim _{n \rightarrow \infty} \sup _{x \in B}\left\|T(h) T\left(s_{n}\right) x-T\left(s_{n}\right) x\right\|=0,
$$


where $B$ is any bounded set containing $\left\{x_{n}\right\}$. Moreover, since $\left\{x_{n}\right\},\left\{T\left(s_{n}\right) x_{n}\right\}$ are bounded, and $\alpha_{n} \rightarrow 0$ as $n \rightarrow \infty$, (3.12) implies that

$$
\limsup _{n \rightarrow \infty}\left(\left\|y_{n+1}-y_{n}\right\|-\left\|x_{n+1}-x_{n}\right\|\right) \leq 0 .
$$

Hence, by Lemma 2.4 we have $\lim _{n \rightarrow \infty}\left\|y_{n}-x_{n}\right\|=0$ since $x_{n+1}-x_{n}=\beta_{n}\left(y_{n}-x_{n}\right)$. Consequently, $\lim _{n \rightarrow \infty}\left\|x_{n+1}-x_{n}\right\|=0$.

It follows from (3.11) that

$$
\begin{aligned}
\left\|x_{n}-T\left(s_{n}\right) x_{n}\right\| \leq & \left\|x_{n}-x_{n+1}\right\|+\left\|x_{n+1}-T\left(s_{n}\right) x_{n}\right\| \\
\leq & \left\|x_{n}-x_{n+1}\right\|+\|\left(1-\beta_{n}\right)\left(x_{n}-T\left(s_{n}\right) x_{n}\right) \\
& +\beta_{n}\left(Q_{K}\left[\alpha_{n}\left(\gamma_{n} x_{n}\right)+\left(1-\alpha_{n}\right) T\left(s_{n}\right) x_{n}\right]-T\left(s_{n}\right) x_{n}\right) \| \\
\leq & \left\|x_{n}-x_{n+1}\right\|+\left(1-\beta_{n}\right)\left\|x_{n}-T\left(s_{n}\right) x_{n}\right\|+\alpha_{n} \gamma_{n}\left\|x_{n}-T\left(s_{n}\right) x_{n}\right\| \\
& \quad+\alpha_{n}\left(1-\gamma_{n}\right)\left\|T\left(s_{n}\right) x_{n}\right\| \\
= & \left\|x_{n}-x_{n+1}\right\|+\left(1-\beta_{n}+\alpha_{n} \gamma_{n}\right)\left\|x_{n}-T\left(s_{n}\right) x_{n}\right\|+\alpha_{n}\left(1-\gamma_{n}\right)\left\|T\left(s_{n}\right) x_{n}\right\| .
\end{aligned}
$$

So,

$$
\left\|x_{n}-T\left(s_{n}\right) x_{n}\right\| \leq \frac{1}{\beta_{n}-\alpha_{n} \gamma_{n}}\left(\left\|x_{n}-x_{n+1}\right\|+\alpha_{n}\left(1-\gamma_{n}\right)\left\|T\left(s_{n}\right) x_{n}\right\|\right) \rightarrow 0
$$

Since

$$
\begin{aligned}
& \left\|x_{n}-T(h) x_{n}\right\| \\
& \quad \leq\left\|x_{n}-T\left(s_{n}\right) x_{n}\right\|+\left\|T\left(s_{n}\right) x_{n}-T(h) T\left(s_{n}\right) x_{n}\right\|+\left\|T(h) T\left(s_{n}\right) x_{n}-T(h) x_{n}\right\| \\
& \quad \leq 2\left\|x_{n}-T\left(s_{n}\right) x_{n}\right\|+\left\|T\left(s_{n}\right) x_{n}-T(h) T\left(s_{n}\right) x_{n}\right\|,
\end{aligned}
$$

from (3.13) and (3.14), we have

$$
\lim _{n \rightarrow \infty}\left\|x_{n}-T(h) x_{n}\right\|=0 .
$$

Notice that $\left\{x_{n}\right\}$ is bounded. Put $x^{*}=Q_{F(S)}(0)$. Then there exists a positive number $R$ such that $B\left(x^{*}, R\right) \cap K$ contains $\left\{x_{n}\right\}$. Moreover, $B\left(x^{*}, R\right) \cap K$ is $T(s)$-invariant for all $s \geq 0$ and so, without loss of generality, we can assume that $\{T(s): s \geq 0\}$ is a nonexpansive semigroup on $B\left(x^{*}, R\right) \cap K$. We take a subsequence $\left\{x_{n_{k}}\right\}$ of $\left\{x_{n}\right\}$ such that

$$
\limsup _{n \rightarrow \infty}\left\langle-x^{*}, j\left(x_{n}-x^{*}\right)\right\rangle=\lim _{k \rightarrow \infty}\left\langle x^{*}, j\left(x_{n_{k}}-x^{*}\right)\right\rangle .
$$

We may also assume that $x_{n_{k}} \rightarrow \tilde{x}$. It follows from Lemma 2.3 and (3.15) that $\tilde{x} \in F(S)$ and hence

$$
\left\langle-x^{*}, j\left(\tilde{x}-x^{*}\right)\right\rangle \leq 0
$$


Since $j$ is weakly sequentially continuous, we have

$$
\limsup _{n \rightarrow \infty}\left\langle-x^{*}, j\left(x_{n}-x^{*}\right)\right\rangle=\lim _{k \rightarrow \infty}\left\langle-x^{*}, j\left(x_{n_{k}}-x^{*}\right)\right\rangle=\left\langle-x^{*}, j\left(\tilde{x}-x^{*}\right)\right\rangle \leq 0 .
$$

Since $\lim _{n \rightarrow \infty}\left\|y_{n}-x_{n}\right\|=0$, we have $y_{n}-x^{*} \rightarrow x_{n}-x^{*}$, so

$$
\limsup _{n \rightarrow \infty}\left\langle-x^{*}, j\left(y_{n}-x^{*}\right)\right\rangle=\limsup _{n \rightarrow \infty}\left\langle-x^{*}, j\left(x_{n}-x^{*}\right)\right\rangle \leq 0
$$

Set $u_{n}=\alpha_{n}\left(\gamma_{n} x_{n}\right)+\left(1-\alpha_{n}\right) T\left(s_{n}\right) x_{n}$. It follows that $y_{n}=Q_{K} u_{n}$ for all $n \geq 0$. By Proposition 2.1(3), we have

$$
\left\langle y_{n}-u_{n}, j\left(y_{n}-x^{*}\right)\right\rangle \leq 0,
$$

and so

$$
\begin{aligned}
\left\|y_{n}-x^{*}\right\|^{2}= & \left\langle y_{n}-x^{*}, j\left(y_{n}-x^{*}\right)\right\rangle \\
= & \left\langle y_{n}-u_{n}, j\left(y_{n}-x^{*}\right)\right\rangle+\left\langle u_{n}-x^{*}, j\left(y_{n}-x^{*}\right)\right\rangle \\
\leq & \left\langle u_{n}-x^{*}, j\left(y_{n}-x^{*}\right)\right\rangle \\
= & \alpha_{n} \gamma_{n}\left\langle x_{n}-x^{*}, j\left(y_{n}-x^{*}\right)\right\rangle-\alpha_{n}\left(1-\gamma_{n}\right)\left\langle x^{*}, j\left(y_{n}-x^{*}\right)\right\rangle \\
& +\left(1-\alpha_{n}\right)\left\langle T\left(s_{n}\right) x_{n}-x^{*}, j\left(y_{n}-x^{*}\right)\right\rangle \\
\leq & \alpha_{n} \gamma_{n}\left\|x_{n}-x^{*}\right\|\left\|j\left(y_{n}-x^{*}\right)\right\|-\alpha_{n}\left(1-\gamma_{n}\right)\left\langle x^{*}, j\left(y_{n}-x^{*}\right)\right\rangle \\
& +\left(1-\alpha_{n}\right)\left\|T\left(s_{n}\right) x_{n}-x^{*}\right\|\left\|j\left(y_{n}-x^{*}\right)\right\| \\
\leq & {\left[1-\left(1-\gamma_{n}\right) \alpha_{n}\right]\left\|x_{n}-x^{*}\right\|\left\|y_{n}-x^{*}\right\|-\alpha_{n}\left(1-\gamma_{n}\right)\left\langle x^{*}, j\left(y_{n}-x^{*}\right)\right\rangle } \\
\leq & \frac{1-\left(1-\gamma_{n}\right) \alpha_{n}}{2}\left\|x_{n}-x^{*}\right\|^{2}+\frac{1}{2}\left\|y_{n}-x^{*}\right\|^{2}-\alpha_{n}\left(1-\gamma_{n}\right)\left\langle x^{*}, j\left(y_{n}-x^{*}\right)\right\rangle,
\end{aligned}
$$

that is

$$
\left\|y_{n}-x^{*}\right\|^{2} \leq\left[1-\left(1-\gamma_{n}\right) \alpha_{n}\right]\left\|x_{n}-x^{*}\right\|^{2}-2 \alpha_{n}\left(1-\gamma_{n}\right)\left\langle x^{*}, j\left(y_{n}-x^{*}\right)\right\rangle .
$$

By the convexity of $\|\cdot\|^{2}$, we have

$$
\begin{aligned}
\left\|x_{n+1}-x^{*}\right\|^{2} & \leq\left(1-\beta_{n}\right)\left\|x_{n}-x^{*}\right\|^{2}+\beta_{n}\left\|y_{n}-x^{*}\right\|^{2} \\
& \leq\left[1-\left(1-\gamma_{n}\right) \alpha_{n} \beta_{n}\right]\left\|x_{n}-x^{*}\right\|^{2}-2\left(1-\gamma_{n}\right) \alpha_{n} \beta_{n}\left\langle x^{*}, j\left(y_{n}-x^{*}\right)\right\rangle .
\end{aligned}
$$

By Lemma 2.5, we conclude that $x_{n} \rightarrow x^{*}$.

Remark 3.4 Theorem 3.3 improves and extends Theorem 3.3 of Yao et al. [7] in the following aspects.

(1) From a real Hilbert space to a reflexive, strictly convex and uniformly smooth Banach space which satisfies Opial's condition.

(2) $\frac{1}{\lambda_{n}} \int_{0}^{\lambda_{n}} T(s) x_{n} d s$ is replaced by $T\left(s_{n}\right) x_{n}$. 


\section{Competing interests}

The authors declare that they have no competing interests.

\section{Authors' contributions}

The main idea of this paper is proposed by XW. All authors read and approved the final manuscript.

Received: 3 June 2013 Accepted: 17 September 2013 Published: 07 Nov 2013

\section{References}

1. Aleyner, A, Censor, Y: Best approximation to common fixed points of a semigroup of nonexpansive operators. J. Nonlinear Convex Anal. 6, 137-151 (2005)

2. Sunthrayuth, $P$, Kumam, $P$ : A general iterative algorithm for the solution of variational inequalities for a nonexpansive semigroup in Banach spaces. J. Nonlinear Anal. Optim. 1, 139-150 (2010)

3. Yao, Y, Liou, Y-C, Yao, J-C: Algorithms for finding minimum norm solution of equilibrium and fixed point problems for nonexpansive semigroups in Hilbert spaces. J. Nonlinear Convex Anal. (in press)

4. Yang, P, Yao, Y, Liou, Y-C, Chen, R: Hybrid algorithms of nonexpansive semigroups for variational inequalities. J. Appl. Math. (2012). doi:10.1155/2012/634927

5. Yao, Y, Cho, YJ, Liou, Y-C: Hierarchical convergence of an implicit double-net algorithm for nonexpansive semigroups and variational inequalities. Fixed Point Theory Appl. 2011, Article ID 101 (2011). doi:10.1186/1687-1812-2011-101

6. Chen, $\mathrm{R}, \mathrm{He}, \mathrm{H}$ : Viscosity approximation of common fixed points of nonexpansive semigroups in Banach space. Appl. Math. Lett. 20, 751-757 (2007)

7. Yao, Y, Kang, Jl, Cho, YJ, Liou, Y-C: Approximation of fixed points for nonexpansive semigroup in Hilbert spaces. Fixed Point Theory Appl. (2013). doi:10.1186/1687-1812-2013-31

8. Gossez, J-P, Lami Dozo, E: Some geometric properties related to the fixed point theory for nonexpansive mappings. Pac. J. Math. 40, 565-573 (1972)

9. Goebel, K, Reich, S: Uniform Convexity, Hyperbolic Geometry, and Nonexpansive Mappings. Dekker, New York (1984)

10. Kopecka, E, Reich, S: Nonexpansive retracts in Banach spaces. Banach Cent. Publ. 77, 161-174 (2007)

11. Reich, S: Asymptotic behavior of contractions in Banach spaces. J. Math. Anal. Appl. 44, 57-70 (1973)

12. Kitahara, S, Takahashi, W: Image recovery by convex combinations of sunny nonexpansive retractions. Topol. Methods Nonlinear Anal. 2, 333-342 (1993)

13. Jung, JS: Iterative approach to common fixed points of nonexpansive mappings in Banach spaces. J. Math. Anal. Appl. 302, 509-520 (2005)

14. Suzuki, T: Strong convergence of Krasnoselskii and Mann's sequences for one-parameter nonexpansive semigroup without Bochner integrals. J. Math. Anal. Appl. 305, 227-239 (2005)

15. Xu, HK: An iterative approach to quadratic optimization. J. Optim. Theory Appl. 116, 659-678 (2003)

10.1186/1687-1812-2013-248

Cite this article as: Wang et al.: Strong convergence theorems for fixed point problems of a nonexpansive semigroup in a Banach space. Fixed Point Theory and Applications 2013, 2013:248

\section{Submit your manuscript to a SpringerOpen ${ }^{\circ}$ journal and benefit from:}

- Convenient online submission

- Rigorous peer review

Immediate publication on acceptance

- Open access: articles freely available online

- High visibility within the field

- Retaining the copyright to your article 